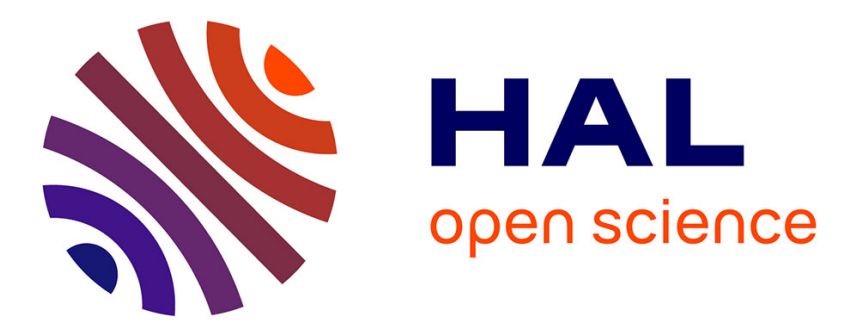

\title{
Le principe de neutralité axiologique et le rapport au savoir scientifique des sociologues. Une exploration compréhensive à partir de l'expérience de cinq professeurs d'un département de sociologie.
}

Pascal Fugier

\section{To cite this version:}

Pascal Fugier. Le principe de neutralité axiologique et le rapport au savoir scientifique des sociologues. Une exploration compréhensive à partir de l'expérience de cinq professeurs d'un département de sociologie.. Spirale - Revue de Recherches en Éducation , 2013. halshs-01653028

\author{
HAL Id: halshs-01653028 \\ https://shs.hal.science/halshs-01653028
}

Submitted on 30 Nov 2017

HAL is a multi-disciplinary open access archive for the deposit and dissemination of scientific research documents, whether they are published or not. The documents may come from teaching and research institutions in France or abroad, or from public or private research centers.
L'archive ouverte pluridisciplinaire HAL, est destinée au dépôt et à la diffusion de documents scientifiques de niveau recherche, publiés ou non, émanant des établissements d'enseignement et de recherche français ou étrangers, des laboratoires publics ou privés. 
Le principe de neutralité axiologique et le rapport au savoir scientifique des sociologues. Une exploration compréhensive à partir de l'expérience de cinq professeurs d'un département de sociologie

Pascal Fugier

\section{Citer ce document / Cite this document :}

Fugier Pascal. Le principe de neutralité axiologique et le rapport au savoir scientifique des sociologues. Une exploration compréhensive à partir de l'expérience de cinq professeurs d'un département de sociologie. In: Spiral-E. Revue de recherches en éducation, supplément électronique au $n^{\circ} 51,2013$. Supplément au $n^{\circ} 51$ : Education et mondialisation. pp. 59-70;

http://www.persee.fr/doc/spira_2118-724x_2013_sup_51_1_1727 


\begin{abstract}
The interviews which we led with five professors of a French department of sociology reveal the dominating place which ethical neutrality has in their relationship to scientific knowledge. Principle of neutrality limits the use and the expression demonstrates of their feelings and affects. However, we can also glimpse the ambiguity of their posture, an ideological wrestling as well as the tracks of a social suffering can show themselves behind the refusal posted of their implication. If the reduced size of the sample and its local demarcation prevent us from clearing ideal types for the Human Sciences, this qualitative research allows nevertheless to glimpse that a relationship to scientific knowledge emancipated to the principle of neutrality can find its place in the sciences of the education.
\end{abstract}

\title{
Résumé
}

Les entretiens menés auprès des cinq professeurs d'un département français de sociologie révèlent la place prépondérante que le principe de neutralité axiologique tend à avoir dans leur rapport au savoir scientifique, limitant l'usage et l'expression manifeste de leurs émotions et affects. Toutefois, la polysémie que recouvre ce principe permet aussi d'entrevoir l'ambiguïté de leur posture, une lutte idéologique ainsi que les traces d'une souffrance sociale pouvant se révéler derrière le refus affiché de leur implication. Si la taille réduite de l'échantillon et sa délimitation locale nous empêchent de dégager des idéaux-types valables pour l'ensemble du champ des SHS, ce travail de recherche qualitatif nous permet néanmoins d'entrevoir qu'un rapport au savoir scientifique émancipé du principe de neutralité axiologique peut y trouver sa place. 


\title{
LE PRINCIPE DE NEUTRALITÉ AXIOLOGIQUE ET LE RAPPORT AU SAVOIR SCIENTIFIQUE DES SOCIOLOGUES
}

\author{
UNE EXPLORATION COMPRÉHENSIVE \\ À PARTIR DE L'EXPÉRIENCE DE CINQ PROFESSEURS \\ D'UN DÉPARTEMENT DE SOCIOLOGIE
}

\begin{abstract}
Résumé : Les entretiens menés auprès des cinq professeurs d'un département français de sociologie révèlent la place prépondérante que le principe de neutralité axiologique tend à avoir dans leur rapport au savoir scientifique, limitant l'usage et l'expression manifeste de leurs émotions et affects. Toutefois, la polysémie que recouvre ce principe permet aussi d'entrevoir l'ambiguïté de leur posture, une lutte idéologique ainsi que les traces d'une souffrance sociale pouvant se révéler derrière le refus affiché de leur implication. Si la taille réduite de l'échantillon et sa délimitation locale nous empêchent de dégager des idéaux-types valables pour l'ensemble du champ des SHS, ce travail de recherche qualitatif nous permet néanmoins d'entrevoir qu'un rapport au savoir scientifique émancipé du principe de neutralité axiologique peut y trouver sa place.

Mots-clés : rapport au savoir, rapport aux valeurs, engagement, passion, subjectivité, sociologie clinique et critique.
\end{abstract}

Dans le cadre de notre thèse de doctorat (Fugier 2010a), nous avons analysé les discours sociologique et autobiographique d'une cohorte de doctorants et jeunes docteurs d'un département de sociologie d'une université française $(n=30)$, accompagnés de leurs directeurs de thèse respectifs $(n=5)$. Notre matériau empirique comprend un corpus d'écrits sociologiques (ouvrages, articles, thèses de doctorat et mémoires de master). Nous avons aussi réalisé un entretien à visée épistémologique et un récit de vie avec douze docteurs en sociologie, trois doctorants ainsi que les cinq professeurs de sociologie.

Nous nous concentrerons ici sur les entretiens effectués auprès des professeurs de sociologie : Barbara, sociologue des professions, interactionniste ; César, sociologue de la connaissance, constructiviste ; Louis, sociologue du crime, structuraliste ; Mélodie, sociologue de la culture, sensible à l'approche critique et postmoderne ; et Modeste, sociologue marxien développant une théorie générale du capitalisme. Durant ces entretiens, nous les avons invités à expliciter leur conception du métier de so-

Spiral-E - Revue de Recherches en Éducation - 2013

Supplément électronique au $N^{\circ} 51$ (59-70) 
ciologue, leur posture d'enseignant-chercheur et les ancrages théoriques et méthodologiques qui fondent l'identité de leur sociologie.

Si ce que nous avançons est avant tout valable dans le microcosme universitaire que nous avons étudié (et ce pour la période 1999-2009), la diversité et la complexité des représentations recueillies peuvent servir d' «analyseurs » (Lapassade 1971) du champ universitaire, en révélant ses oppositions structurales mais aussi ses ambiguïtés et contradictions. La conduite d'entretiens compréhensifs (Fugier 2010b) constituant un « analyseur construit », apte à « révéler le non-dit de l'institution » sociologique (Lourau 1970, 283).

Afin d'explorer la construction biographique de l'épistémologie et du rapport au savoir des enseignants-chercheurs en sociologie, notre canevas d'entretien se déclinait en différentes thématiques abordant la place qu'ils donnent au travail du concept et à la philosophie (Fugier 2013c), la manière dont ils se confrontent à la tension paradigmatique opposant le déterminisme et la liberté (Fugier 2008b) et plus fondamentalement la manière dont peuvent s'imbriquer à leurs terrains de recherche leur «terrain biographique » et leur «terrain socio-historique» (Fugier 2008a, 2013a). Nous souhaitons revenir ici sur une autre dimension caractéristique de toute démarche intellectuelle, à savoir la construction biographique de leur rapport au savoir scientifique dans sa dimension axiologique. Dimension dont la principale tension voit s'opposer les positions antithétiques de l'engagement et de la distanciation, et auxquelles vont se corréler d'autres oppositions comme celle de la posture critique et de la neutralité axiologique.

Bien que l'échantillon d'étude soit de taille réduite, se limitant à cinq professeurs de sociologie, la conduite des entretiens révèle la polysémie que recouvre le principe de neutralité axiologique (première partie), pourtant pierre angulaire de nombre de cours magistraux et ouvrages pédagogiques de sociologie. Le commentaire d'un extrait de Pierre Bourdieu dans lequel ce dernier entrevoit un lien entre sa colère et sa lucidité leur permet d'interroger la place que les émotions et affects ont dans leur réflexivité (deuxième partie). C'est alors en interrogeant non seulement la possibilité de la neutralité axiologique mais sa nécessité (troisième partie) qu'un rapport au savoir scientifique d'orientation critique et clinique transparaît parmi certains enseignants-chercheurs en sociologie.

\section{LA POLYSÉMIE DE LA NEUTRALITÉ AXIOLOGIQUE}

Lorsque nous avons abordé la question de la neutralité axiologique aux professeurs de sociologie de notre échantillon d'étude, nous avons pu en recueillir des significations différentes. Ainsi, Mélodie associe la neutralité axiologique du sociologue à la rupture épistémologique, soit à la rupture avec ses prénotions et le sens commun. Elle évoque le «vrai travail» de rupture qu'elle effectue dès qu'elle entame une recherche, en considérant qu'il s'agit de quelque chose de transitoire et qu'il faut inlassablement recommencer.

Mélodie se distingue de Louis, qui conçoit la neutralité axiologique comme une posture professionnelle plutôt qu'un travail interminable. À ce propos, Louis nous raconte comment il a attrapé le «troisième oeil» à l'âge de 11 ans, à travers l'expérience de l'internat au sein d'un collège prestigieux et qu'il assimile à une «déportation »: 
"J'ai attrapé le troisième oeil à 11 ans, à travers cette rupture, ça a été une vraie rupture... C'est comme ça à mon avis, j'avais déjà l'œil (du sociologue, la rupture...) à ce moment-là. Je pense que ça peut être un élément d'interprétation important... [...] c'est là que je suis devenu anthropologue [...] moi j'étais un petit con, le petit fils de commerçant là-dedans. Et... donc c'était un bouleversement culturel hallucinant pour moi, mais vraiment... J'ai passé huit jours à pleurer toutes les larmes de mon corps... J'étais "déporté » dans un collège de luxe. Et... à ce moment-là, j'ai vu les choses autrement...j'étais comme un Indien qui arrivait ailleurs...»

Véritable rupture biographique pour ce «fils de commerçant» qui se retrouve entouré de camarades issus de la bourgeoisie, Louis situe dans l'expérience de l'internat la genèse d'un habitus d'anthropologue ${ }^{1}$, en tant que principe générateur d'une disposition à la curiosité, au désintéressement et à l'écoute bienveillante envers toutes les choses qui font le monde, même celles qui paraissent immondes pour le sens commun (il est sociologue du crime et s'est notamment intéressé aux bourreaux). Louis se situe donc plutôt dans un positionnement axiologique affirmant l'éradication durable des présupposés de sens commun, plutôt que leur éternel retour nécessitant un interminable travail de neutralisation.

Mais si Mélodie s'évertue à neutraliser ses présupposés quand elle «travaille sur le terrain » et l'analyse, elle estime par contre que la neutralité axiologique n'est pas souhaitable à l'amont d'un travail de recherche. L'élaboration de l'objet de sa recherche doit demeurer un véritable «engagement» :

«Le choix [d'objet] pour moi c'est un engagement. [Mes objets de recherche] correspondent tous à une volonté que j'avais de démontrer quelque chose. J'avais un objectif derrière ma recherche. Je n'ai jamais pris une recherche pour faire une recherche. Alors bon, là, je dis non. Par contre à partir du moment où j'ai décidé de faire une recherche, la rupture, il faut qu'elle soit là.»

Une question se pose : que fait-elle du fameux «quelque chose» qu'elle veut « démontrer»? Est-ce qu'il est éradiqué à l'occasion de son travail de rupture ? Et, dans ce cas, cela signifie que l'intérêt de ce «quelque chose » se limite simplement à donner une impulsion à la recherche du sociologue et la décide à explorer tel ou tel fragment de réalité. Ou bien est-ce que ce «quelque chose» l'accompagne durant tout son procès de travail, mais en étant mis sous contrôle par un travail de rupture épistémologique qui le réduit au statut de simple piste de recherche ou d'hypothèse ?

Il apparaît que Mélodie distingue son identité personnelle, terreau des présupposés qui sont à l'impulsion de ses recherches, et son identité professionnelle de sociologue, exigeant leur bannissement. Et si elle reconnaît que ce qu'on est «individuellement » résiste à ce travail de rupture et fait intrusion dans la recherche à l'insu du sociologue, elle exige un tel clivage identitaire en tant que directrice de thèse. Ainsi, quand elle repère des présupposés dans le travail sociologique de l'un de ses doctorants, elle ne les perçoit pas comme ce qui peut orienter son regard vers un fragment de réalité qui serait resté sans cela invisible ou lettre morte :

\footnotetext{
${ }^{1}$ Bien que professeur de sociologie et titulaire d'une thèse en sociologie, Louis se considère davantage comme un anthropologue (du crime en l'occurrence), comme il me signale durant notre entretien, Marcel Mauss à l'appui : "disons, sans vouloir m'abriter derrière des idoles... Mauss le dit, il dit que dans toutes ces divisions entre disciplines, il dit que ce ne sont que des clivages académiques, mais pas par rapport à l'objet, entre histoire, sociologie, ethnologie, linguistique etc., etc. Et Mauss lui-même dit... il faudrait résumer sous le terme d'anthropologie l'ensemble de cette approche diversifiée. »
} 
«Hector, mon dieu le pauvre, il en a eu... Je rouspétais par moments, parce qu'alors il a des présupposés pas possibles... Parce que... Par exemple par rapport à son terrain, à ses entretiens, dans sa façon d'analyser, comme il a des présupposés, bien il est en train de glaner ce qui va alimenter ses présupposés, et donc... s'il n'y a pas de rupture...»

Lorsque nous avons posé la question de la neutralité axiologique à Barbara, elle n'a pas évoqué l'impératif de rupture épistémologique mais celui du contrôle des pairs. Face à la porosité de la frontière séparant "l'homme ordinaire » de l'homme savant, le sociologue doit suffisamment expliciter son modus operandi, afin que la communauté des chercheurs soit en capacité de falsifier ses assertions :

«[...] il faut donner aux lecteurs la capacité de critiquer ce que tu as vu, les entretiens, combien tu en as fait... C'est-à-dire que, il faut que la méthode soit contrôlable, et ce pas que par toi, mais par autrui aussi. Je suis absolument convaincu que ça, c'est la seule manière de s'en sortir en sociologie. »

Plutôt que de renvoyer l'impossibilité de la neutralité axiologique à l'individualité du sociologue (Mélodie) et son ordinarité (Barbara), Modeste insiste sur le fait que c'est la structure même de la réalité sociale, et en l'occurrence sa structure conflictuelle, qui rend la neutralité axiologique impossible. Ce qui correspond davantage à la situation socio-historique de l'enseignant-chercheur plutôt qu'à son histoire de vie :

«Non, on ne peut pas être neutre. On est toujours dans une certaine situation [...] qui est, à des degrés divers et selon des médiations multiples, une situation de conflits... et on est amené à prendre position dans ces conflits. »

De la même manière, lorsque nous introduisons la question de la neutralité axiologique à César, il nous fait remarquer sur un ton amusé que «pendant la guerre, les Suisses étaient neutres...». Ce faisant, César insinue que la neutralité est ellemême une prise de position.

Pour résumer, on peut avancer que Mélodie et Barbara insistent sur la limite dispositionnelle du sociologue dans sa quête de neutralité axiologique (il ne peut être totalement en rupture par rapport à ce qu'il est en tant qu'homme ordinaire), tandis que Modeste et César insistent davantage sur sa limite positionnelle (il ne peut pas se situer au-delà ou en dehors de la situation socio-historique qu'il s'attache à étudier). Louis est le seul professeur de l'échantillon pour qui la neutralité axiologique est quelque chose de possible. Il s'agit selon lui d'une disposition durable incorporée sous la forme d'un habitus, permettant au sociologue d'être "complètement extérieur », un véritable étranger parmi ses semblables. À l'inverse, Modeste affirme que l'impératif auquel doit se soumettre le sociologue consiste à prendre conscience de son parti pris et d'essayer d'en repérer les limites et effets pervers :

"Il faut absolument se convaincre que l'on est toujours de parti pris, donc, prendre conscience de son parti pris et essayer de repérer les effets d'aveuglement ou les effets d'illusion que ces partis pris font naître... Et, c'est là que l'on a besoin des uns et des autres, c'est-à-dire qu'on a vraiment besoin d'écouter et de lire des gens qui travaillent à partir d'autres points de vue, donc d'autres paradigmes, d'autres méthodes, d'autres engagements que soi. Et de les prendre au sérieux... mais de les lire...»

Il est intéressant de noter que si Modeste se réfère tout comme Barbara aux bénéfices du cadre collectif au sein duquel s'inscrit le sociologue afin de repérer ses 
partis pris, ils ne se réfèrent pas exactement au même type d'interlocuteurs : il s'agit avant tout d'interactions entre lui et des livres pour Modeste, tandis qu'il s'agit avant tout de collègues pour Barbara. L'importance accordée au contrôle par les livres pour l'un, au contrôle par les pairs pour l'autre, témoigne de la construction de deux différents rapports au savoir scientifique, Modeste considérant explicitement durant son entretien que le premier terrain du sociologue est « sa bibliothèque », tandis que Barbara enracine le métier de sociologue dans la pratique du «terrain».

\section{TAIRE LA PASSION ET L'ENGAGEMENT}

\section{DE L'ENSEIGNANT CHERCHEUR}

Afin que les professeurs de sociologie interrogés explicitent davantage leur positionnement relatif au principe de neutralité axiologique, nous leur avons proposé de commenter un extrait de Pierre Bourdieu dans lequel il fait part de son rapport critique aux valeurs. Mais plutôt que de l'identifier comme un biais ou une limite heuristique, il l'élève en condition de possibilité de sa lucidité. Celui qui est pourtant un des auteurs du Métier de sociologue, indique combien son implication affective à l'égard de certaines réalités ont contribué à sa relative lucidité, sans que ce soit pour autant antinomique avec ses efforts de distanciation et sa vigilance à l'égard de sa propre subjectivité :

«Ce que je dis là est à peine articulé, mais je vois un lien entre mon impatience, mon énervement, ma révolte, mon indignation et la «lucidité »- je ne sais pas comment appeler ça. Le travail scientifique, cela ne se fait pas avec des bons sentiments. Ça se fait avec des passions ; après il faut les contrôler. [...] Pour travailler, il faut être en colère. Mais la colère ne suffit pas. Il faut aussi travailler pour contrôler sa colère » (Bourdieu 2000).

En réponse à cet extrait, Mélodie soutient que le sociologue doit être effectivement en colère, mais en la restreignant à la phase du choix d'objet. Son positionnement axiologique demeure cohérent et la colère du sociologue, tout comme ses présupposés, ne doivent pas traverser l'ensemble du procès de recherche, mais seulement l'initier.

La réponse de Barbara est intéressante parce qu'ambiguë : d'un côté, elle se montre très réservée à l'idée de faire de la sociologie avec colère ou plus largement dans une forme d'engagement qui va au-delà du principe tautologique de la quête du « savoir pour le savoir ». Et cette réserve, elle l'impose particulièrement à ses doctorants (tout comme Mélodie), qui ne doivent pas travestir le sens de leur recherche en leur donnant une signification et une direction militante ou critique. Mais, d'un autre côté, elle se demande si cette réserve et le soin qu'elle prend à ne pas donner des signes ostentatoires d'une telle implication ne se fondent pas justement sur le fait que sa sociologie est profondément engagée :

"C'est compliqué cette question-là [la question de la colère, de la passion, des "mauvais sentiments" du sociologue], parce que, en même temps, moi je fais vachement gaffe, peut-être parce que d'une certaine manière je fais de la sociologie engagée, mais du coup, je fais gaffe, je fais extrêmement gaffe avec les étudiants, à leur dire que le premier objectif c'est d'augmenter le stock des connaissances, il s'agit bien de comprendre mieux ce qui se passe. Sachant qu'il s'agit de mieux comprendre avec des gens, il ne s'agit pas de mieux comprendre ma propre vie. Donc, en même 
temps, pour ne pas être emportées par ses passions, ça m'oblige à dire "attention", on comprend d'abord et après ça peut être utilisé par les groupes sociaux en question... [...] Je trouve que là-dessus, c'est très important d'avertir les étudiants que la connaissance, c'est la connaissance. Qu'après, qu'il y ait des professionnels qui s'emparent des résultats scientifiques pour en faire ce qu'ils veulent, on est dans le monde du politique. Mais moi je ne veux pas des thèses politiques, je veux des thèses scientifiques.»

La prise de position de Barbara est complexe. Si elle pose une frontière apparemment étanche entre le politique et le scientifique, on pressent aussi que sa sociologie constitue une forme de combat idéologique. Malheureusement, elle ne nous dit rien quant à l'identité de sa "sociologie engagée ». Néanmoins, nous pouvons nous référer à d'autres extraits de son entretien, dénotant une composante plus biographique, lorsqu'elle critique le stigmate de «l'idiot culturel » dont sont victimes les agriculteurs, étant elle-même fille de fermier. L'observation participante que nous avons pu mener en tant qu'étudiant dans l'université étudiée nous permet d'affirmer combien cette critique a une place importante dans son discours, mais aussi plus largement dans son vécu.

Nous pensons notamment ici à une scène qui s'est déroulée lorsque nous étions inscrit en deuxième année de master. Barbara avait organisé un tour de table à l'occasion d'une séance de travaux dirigés en nous demandant de présenter brièvement nos projets de recherche. C'est alors qu'un des étudiants de la promotion s'est montré profondément dédaigneux à l'égard des "sociologues de terrain " (identité à laquelle est très attachée Barbara), promouvant à l'inverse toute l'excellence des théoriciens, qui travaillent sur des sujets « sérieux» comme le pouvoir (Modeste, en tant que sociologue du politique, et César, en tant que sociologue de la connaissance, suscitent tout son intérêt). Barbara lui fit remarquer que plusieurs sociologues lui collaient l'étiquette de «sociologue des vaches » (du fait qu'elle a beaucoup travaillé sur la profession agricole) et qu'elle assumait totalement cette étiquette. L'étudiant ne put alors s'empêcher de s'esclaffer, tant ce champ d'étude lui paraissait ridicule. Si Barbara parvint alors à se contenir puis changea de sujet, nous avons été sensible à son regard et à sa posture corporelle, trahissant combien elle pouvait être émue du discrédit que cet étudiant portait à son égard. Nous pensons que ces éléments d'observation donnent un sens à la « sociologie engagée » dont Barbara ne nous parle qu'à demimot dans le cadre de l'entretien. Car c'est toujours la figure de «l'idiot culturel » qui se joue dans ces interactions.

Les propos de Louis réservent aussi leur part de mystère et d'ambiguïté. Car si Louis affirme atteindre la si convoitée neutralité axiologique, s'il déclare ne pas engager la moindre passion ou la moindre colère dans ses recherches, se limitant à "l'exigence d'un travail bien fait... une sorte de disponibilité, de posture d'ouverture », il reconnaît toutefois que ce que dit Pierre Bourdieu dans l'extrait précédemment cité «n'est pas faux», mais il précise immédiatement que ce n'est pas comme cela que lui « vit » la recherche sociologique. Louis reconnaît une place à la subjectivité du sociologue mais en la situant en amont de la recherche : de la même manière que Mélodie avance qu'on ne fait jamais de la sociologie sur tel ou tel objet «par hasard», Louis précise qu'on devient sociologue parce qu'on n'est pas un «imbécile heureux». Si on se dirige vers la sociologie, c'est bien parce qu'on n'est pas satisfait de la société qui nous environne. Mais Louis revient alors immédiatement dans l'ordre 
de son discours (Foucault 1971) et explique que ce mécontentement amène justement le sociologue à prendre ses distances par rapport au monde et le conduit, d'une certaine manière, à ne plus l'habiter. Ce qui présuppose donc que dès l'entame de sa recherche, la subjectivité et les affects du sociologue n'ont plus leur place. Seule la distance objectivante est présente et légitime :

"Ce qui vous amène à avoir une posture que j'appellerais intellectuelle, c'est parce que vous n'êtes pas satisfaits du monde, sinon vous baignez dans le monde, à $37^{\circ}$ comme un imbécile heureux et... Je veux dire, la réflexion elle commence quand vous en sortez, quand vous vous mettez à distance, et vous vous mettez à distance parce que... bon, si on était satisfait, à mon avis, on ne prendrait pas conscience... La conscience, d'une certaine façon, elle est toujours un peu malheureuse. Il y a un arrachement d'une certaine façon...»

Nous souhaitons insister sur l'ambiguïté du discours de Louis. Car si la souffrance sociale que peut générer une institution totale comme l'internat (pour nous référer au vécu de Louis) peut conduire l'individu à se mettre à distance de la réalité sociale, condition sociale de sa lucidité, en même temps Louis entrevoit le revers de la médaille de cette lucidité, qui à son tour provoque une souffrance. La souffrance apparaît alors à la fois condition et effet de la lucidité du sociologue. C'est alors que la parole de Louis fait apparaître une dimension identitaire jusqu'alors absente, celle de "l'homme ordinaire », qui est blessé, énervé par la réalité sociale qui l'entoure (alors que «l'homme savant » n'est que pure extériorité, totalement indifférent à l'égard de la réalité sociale). Et c'est un autre système de justification qui rentre en scène, Louis avançant que s' " il y a de quoi » être blessé et en colère face à cette réalité sociale, en prenant alors pour cible l'attitude des bureaucrates et plus largement des «gens de pouvoir », en aucun cas cette colère ne doit s'extérioriser. Non pas parce qu'elle remettrait en cause la lucidité du sociologue mais parce que sinon, il «pèterait les plombs à coup de révolvers » et ferait «tout sauter»! Nous ne sommes donc pas très loin de la réserve que Barbara s'impose et impose à ses doctorants vis-à-vis de la question de l'engagement, de crainte que la passion l'emporte sur la raison. Ici, la parole de Louis se veut plus explicite quant au danger de la passion : c'est la mort d'autrui. Alors, afin d'éviter de succomber à de telles pulsions meurtrières, il faut bien se garder d'être affecté, «blessé, énervé » par le réel. Pour cela, il vaut mieux être mort ("moi personnellement, comme je suis déjà mort, je m'en fous complètement! ») et prendre ensuite le masque, la persona du sociologue. Telle est la radicale option que Louis a adopté, pour ne pas «tout faire sauter » :

Louis : "je cite toujours cette formule de René Char, il dit "la lucidité est la blessure la plus proche du soleil"... C'est magnifique, c'est superbe. Et bien c'est ça... parce que je pense qu'il était très lucide Bourdieu, extrêmement lucide, et à mon avis il était blessé. Et il y a de quoi. Et c'est vrai que moi aussi je suis absolument blessé et énervé de voir comment ces bureaucrates, administratifs, gens de pouvoir, sont en train de gérer les trucs, mais bon... je veux dire... comme j'ai fait un certain chemin, je ne suis pas un gamin, etc., je ne vais pas m'énerver là-dessus, sinon je prendrais un pétard, je ferais tout sauter. Vous voyez ce que je veux dire... Donc... Non... Et donc je joue au professeur de sociologie...

$\mathrm{PF}$ : Parce que l'énervement que vous pouvez ressentir à titre personnel, vous ne l'utilisez pas comme combustible dans le cadre de vos recherches? 
Louis : Non, ce n'est pas un moteur. Non... Je pense même que c'est nocif. Je pense que ça empoisonne.

PF : Oui, c'est plus un poison qu'un combustible.

Louis : Voilà... Moi je pense que ce qui fait vraiment avancer la machine, c'est d'avancer tout seul, c'est d'ouvrir les yeux, tout simplement, c'est de...d'être en position d'être ouvert, comme ça... Et comme les choses bougent d'elles-mêmes, on est pris, on observe, on capte, et puis après on synthétise...»

Selon Louis, la passion peut donc tuer autrui et empoisonner l'homme savant. Alors il vaut mieux dans ces conditions bien se garder de l'extérioriser. Mais il est frappant de voir à quel point Louis peut se montrer cynique à l'égard du métier de sociologue ("je joue au professeur de sociologie», plutôt que de flinguer tout le monde) et en même très enchanté : la sociologie, c'est la lucidité, c'est l'ouverture, mais au prix de la disparition de l'homme ordinaire. Le sociologue, c'est la sociologie faite homme, destituée de toute subjectivité : c'est «la machine».

En résumé, si le commentaire de l'extrait de Pierre Bourdieu confirme la place prépondérante que le principe de neutralité axiologique peut avoir dans le rapport au savoir scientifique du sociologue (Mélodie, Barbara et Louis tendent de prime abord à restreindre le rôle des passions et des affects à la phase du choix de l'objet), certains de leurs commentaires révèlent une certaine ambiguïté de leur positionnement. On peut ainsi se demander avec Barbara si le refus de manifester toute forme d'engagement dans son travail de recherche et son enseignement ne vise pas à cacher un engagement latent, voire un véritable combat idéologique (à l'encontre de la stigmatisation du monde rural par exemple). De même, si la souffrance sociale de l'enseignant-chercheur apparaît comme ce qui participe de son désir de savoir, la position d'extériorité que manifeste le sociologue peut constituer une «stratégie de défense " (Dejours 2000) visant à échapper à cette souffrance. Le fait que le savoir puisse masquer une souffrance témoigne alors d'un rapport contrarié au savoir scientifique.

Certains sociologues, comme Modeste, sortent de cette ambiguïté en défendant un rapport au savoir scientifique émancipé du principe de neutralité axiologique. Ce que nous allons désormais présenter, en prenant aussi appui sur quelques représentants de la sociologie critique et clinique.

\section{UNE NEUTRALITÉ AXIOLOGIQUE NON SOUHAITABLE}

Parmi les professeurs que nous avons interrogés, Modeste est celui chez qui l'engagement et les partis pris idéologiques (en l'occurrence ses prises de position critiques envers la vision du monde capitaliste) traversent littéralement toute l'œuvre intellectuelle. Alors que Louis conçoit la science comme étant plutôt l'antinomie de l'engagement et du parti pris, Modeste conçoit le parti pris comme une condition de possibilité de la science, et non pas simplement dans sa dimension psychologique (i.e. comme désir de persévérer dans ses efforts d'objectivation) mais dans sa portée proprement heuristique :

«S'il n'y avait pas de parti pris, pourquoi est-ce qu'on ferait de la science? Si quelque part la science était une entreprise complètement désintéressée, sans intérêt, il n'y aurait pas de science... Il faut quand même que celui qui se lance dans une entreprise scientifique soit mu par un solide désir de... un intérêt puissant, qui le mo- 
tive, qui le soutienne... et qui d'ailleurs, au-delà de cet aspect tout à fait subjectif ou même quasiment psychologique de la recherche, va l'amener à se poser un certain nombre de questions qu'il ne se poserait pas s'il n'y avait pas cet intérêt...»

On voit bien ici que Modeste fait «entrer» l'engagement du sociologue dans son travail d'objectivation, alors que Mélodie, Barbara et Louis s'obligent à le "faire sortir", associant l'objectivité et la lucidité du sociologue à sa neutralité. Et lorsque nous lui proposons l'extrait de Pierre Bourdieu, Modeste s'y associe, soulignant toutefois que le propos de l'auteur n'a rien d'extraordinaire, et qu'il s'agit plutôt d'une «banalité » pour quiconque a « réfléchi un tant soit peu à la manière dont on produit une connaissance, c'est-à-dire dont un sujet constitue un objet...». Nous retrouvons ici une référence implicite à la révolution copernicienne kantienne dans la théorie de la connaissance : un objet d'étude ne repose pas sur son objectivité mais se construit à partir des objectivations du sujet (ici le sociologue). Or, ces objectivations, aussi méthodiques qu'elles puissent être, s'opèrent à partir du « rapport aux valeurs » (Weber 1992) et des «intérêts de connaissance » (Habermas 1976) du sujet. Valeurs et intérêts qui se traduisent notamment en affects, en passion.

Si Louis conçoit la passion du sociologue comme un poison, Modeste considère au contraire qu'elle est un combustible que le travail de recherche va consommer afin d'avancer. L'obligation du sociologue à l'égard de sa passion ne consiste donc pas à l'exclure de son procès de recherche (ou l'arrêter à la porte de la recherche, au moment du choix de l'objet) mais à la «travailler » (tel un moteur). Plutôt que de dénier ou refouler sa passion, il faut plutôt chercher à ne pas en être le sujet mais l'auteur :

"Hegel dit ça: "Rien de grand ne se fait sans passion"... Il est évident que c'est le moteur même de l'action subjective, le moteur même du sujet. Le problème c'est que, on sait aussi que la passion peut être aveuglante quoi. Il faut essayer de la contrôler. Mais ce n'est certainement pas dans la dénégation qu'on la contrôlera. C'est-à-dire que... En ce sens-là, je suis tout à fait un spinozien ou un nietzschéen, c'est-à-dire qu'il faut travailler la passion pour lui faire produire quelque chose, dont elle est capable, qu'elle porte en elle-même, mais qu'elle ne produira pas nécessairement... Et ce n'est pas dans la répression ou la dénégation de la passion qu'on s'en rendra maître et surtout qu'on en tira bénéfice quoi. »

Au-delà du microcosme universitaire étudié, nous retrouvons un certain «air de famille » entre le discours de Modeste et le positionnement critique de la sociologie clinique à l'égard de la posture de neutralité axiologique et de la prétention de certains sociologues à être totalement extérieurs vis-à-vis de leur objet d'étude : «Cette position est illusoire, sinon mystificatrice. Le chercheur [...] quoi qu'il fasse, il est partie prenante du monde social et ne peut prétendre à une pure extériorité eu égard à son terrain de recherche » (de Gaulejac et Roche 2007, 14). Nous pouvons alors considérer la neutralité affichée du sociologue comme une prise de position idéologique. Elle est l'indice d'un engagement non avoué, comme nous le laisse entendre César lorsqu'il nous rappelle que la Suisse était «neutre » durant la Seconde Guerre Mondiale... Ce que n'a pas non plus manqué de souligner un des principaux représentants de l'École de Francfort, Theodor W. Adorno, affirmant que la neutralité du chercheur en sciences sociales constitue un véritable acte politique : «Tout comme l'apathie politique, la neutralité scientifique s'avère un acte politique quant à son contenu social. 
Depuis Pareto, le scepticisme positiviste s'accommode du pouvoir existant, même celui de Mussolini » (Adorno et Popper 1979, 30).

Pour notre part, il nous semble important de distinguer la posture de neutralité du sociologue et son travail d'objectivation. En effet, si le sociologue se doit d'effectuer un travail méthodique du concept, de mener méthodologiquement son travail de «terrain » et de se plier autant que possible à l'exercice de la socioanalyse (Fugier 2013b), il n'est pas tenu à la neutralité, c'est-à-dire au renoncement de toute évaluation subjective à l'égard de la réalité étudiée. L'implication idéologique et subjective du sociologue au sein de son travail de recherche apparaît alors à la fois comme limite et potentialité heuristique. Ce qui donne à la rupture épistémologique un caractère ambivalent: "Souvent, les implications éthiques et politiques du sociologue ont avant tout été considérées comme des “obstacles épistémologiques", c'est-à-dire comme des entraves à la scientificité de ses analyses, en le pourvoyant par avance en fausses évidences nées du "sens commun". Aujourd'hui, on est peut-être en mesure d'envisager leur ambivalence : toujours obstacles épistémologiques dont le chercheur doit se méfier à un bout de la chaîne, mais également stimulant cognitif à un autre bout » (Corcuff 2004, 191).

\section{CONCLUSION}

Notre problématique de recherche interroge notamment les liens qui peuvent se nouer entre le rapport aux valeurs d'un enseignant-chercheur et son rapport au savoir scientifique. Malgré les résistances que peuvent manifester les cinq professeurs de sociologie que nous avons interrogés, il apparaît que leurs prises de position théoriques et méthodologiques engagent nécessairement leur subjectivité. Cette dernière pouvant s'afficher sous la forme de valeurs qui vont orienter (en tant qu'idées de valeur culturelles, pour reprendre la terminologie wébérienne) ou évaluer (en tant que jugements de valeur) la portion de réalité singulière que le sociologue a sélectionnée parmi la réalité empirique étudiée. Ce travail de recherche nous conduit alors à poser l'hypothèse que la neutralité axiologique de chaque sociologue est impossible, entendue comme mise en suspens de ses valeurs mais aussi de ses «intérêts de connaissance » (Habermas 1976), et ce durant l'ensemble de son procès de travail. Ce que nous souhaitons désormais approfondir dans le cadre d'une « clinique du rapport au savoir» scientifique (Beillerot, Blanchard-Laville et Mosconi 1996), en étudiant d'autres départements français de sociologie mais aussi d'autres disciplines universitaires, à commencer par les sciences de l'éducation. De quoi inaugurer une approche comparative apte à dégager des idéaux-types représentatifs des différentes formes de rapports au savoir scientifique en vigueur au sein des SHS.

Pascal FUGIER

Université Charles-de-Gaulle - Lille 3

Chercheur associé au Laboratoire de Changement Social et Politique de l'Université Paris Diderot pascal-fugier@orange.fr 
Abstract : The interviews which we led with five professors of a French department of sociology reveal the dominating place which ethical neutrality has in their relationship to scientific knowledge. Principle of neutrality limits the use and the expression demonstrates of their feelings and affects. However, we can also glimpse the ambiguity of their posture, an ideological wrestling as well as the tracks of a social suffering can show themselves behind the refusal posted of their implication. If the reduced size of the sample and its local demarcation prevent us from clearing ideal types for the Human Sciences, this qualitative research allows nevertheless to glimpse that a relationship to scientific knowledge emancipated to the principle of neutrality can find its place in the sciences of the education.

Keywords : relationship to knowledge, relationship to values, implication, passion, subjectivity, clinical and critical sociology.

\section{Bibliographie}

Adorno T. \& Popper K. (1979) De Vienne à Francfort, la querelle allemande. Paris : Éditions Complexe.

Beillerot J., Blanchard-Laville C. \& Mosconi N. (1996) Pour une clinique du rapport au savoir. Paris : L'Harmattan.

Bourdieu P (2000) «A contre pente », entretien réalisé avec P. Mangeot, S. Grelet, V. Patouillard et J. Revel - Vacarme.

URL : http://vacarme.eu .org/article224.html.

Corcuff P. (2004) «Sociologie et engagement : nouvelles pistes épistémologiques » — in : B. Lahire (dir.) A quoi sert la sociologie? (175-194). Paris : La Découverte.

Dejours C. (2000) Souffrance en France. La banalisation de l'injustice sociale. Paris : Le Seuil.

Foucault M. (1971) L'ordre du discours. Paris : Gallimard.

Fugier P (2008a) «Les discours et les terrains des sociologues » - ¿Interrogations? 7.

URL : http://revue-interrogations.org/article.php?article=142

Fugier P (2008b) « Sociologies et déterminismes »- ¿Interrogations? 7.

URL : http://revue-interrogations.org/article.php?article=153

Fugier P. (2010a) Dits et écrits de sociologues et d'apprentis sociologues. Histoires de vie et prises de position sociologiques. Thèse de doctorat en sociologie soutenue à l'Université de Franche-Comté.

Fugier P. (2010b) « Les approches compréhensives et cliniques des entretiens sociologiques »- ¿ Interrogations? 11.

URL : http://www.revue-interrogations.org/Les-approches-comprehensives-et

Fugier P. (2013a) « Le terrain biographique du sociologue comme objet d'étude sociologique et comme support socioanalytique » - in : C. Niewiadomski et C. Delory Momberger (dir.) Territoires contemporains de la recherche biographique. Paris : Téraèdre.

Fugier P. (2013b) «La position de la clinique dans la sociologie de Pierre Bourdieu» - in : V. de Gaulejac, F. Giust-Desprairies et A. Massa (dir.) La recherche clinique en sciences sociales (43-56). Toulouse : Erès.

Fugier P. (2013c) «La place déniée de la philosophie chez les sociologues contemporains : entre lutte des places et coût de l'excellence » — in : J. Deniot, J. 
Réault et L. Delmaire (dir.) Sciences sociales et Humanités. Nantes : Lestamp-Éditions.

Gaulejac V. de et Roche P. (2007) «Introduction» - in : V. de Gaulejac, F. Hanique et P. Roche (dir.) La sociologie clinique. Paris : Erès.

Habermas J. (1976) Connaissance et intérêt. Paris : Gallimard.

Kaufmann J-C. (1996) L'entretien compréhensif. Paris : Nathan.

Lapassade G. (1971) L'arpenteur. Paris : Epi.

Lourau R. (1970) L'analyse institutionnelle. Paris : Minuit.

Weber M. (1992) Essais sur la théorie de la science. Paris : Plon/Pocket. 